\title{
Radiation-induced signaling pathways that promote cancer cell survival (Review)
}

\author{
ASHLEY L. HEIN, MICHEL M. OUELLETTE and YING YAN \\ Eppley Institute for Research in Cancer and Allied Diseases, \\ University of Nebraska Medical Center, Omaha, NE, USA
}

Received June 18, 2014; Accepted August 1, 2014

DOI: $10.3892 /$ ijo.2014.2614

\begin{abstract}
Radiation therapy is a staple cancer treatment approach that has significantly improved local disease control and the overall survival of cancer patients. However, its efficacy is still limited by the development of radiation resistance and the presence of residual disease after therapy that leads to cancer recurrence. Radiation impedes cancer cell growth by inducing cytotoxicity, mainly caused by DNA damage. However, radiation can also simultaneously induce multiple pro-survival signaling pathways, such as those mediated by AKT, ERK and ATM/ATR, which can lead to suppression of apoptosis, induction of cell cycle arrest and/or initiation of DNA repair. These signaling pathways act conjointly to reduce the magnitude of radiation-induced cytotoxicity and promote the development of radioresistance in cancer cells. Thus, targeting these pro-survival pathways has great potential for the radiosensitization of cancer cells. In the present review, we summarize the current literature on how these radiation-activated signaling pathways promote cancer cell survival.
\end{abstract}

\section{Contents}

1. Introduction

2. HER (also called ERBB or EGFR) signaling

3. Extracellular signal-regulated kinase (ERK1/2) pathway

4. AKT signaling pathway

5. Cell cycle checkpoint signaling

Correspondence to: Dr Ying Yan, Eppley Institute for Research in Cancer and Allied Diseases, University of Nebraska Medical Center, 986805 Nebraska Medical Center, Omaha, NE 68198-6805, USA

E-mail: yyan@unmc.edu

Dr Michel M. Ouellette, Eppley Institute for Research in Cancer and Allied Diseases, University of Nebraska Medical Center, 985950 Nebraska Medical Center, Omaha, NE 68198-5950, USA

E-mail:mouellet@unmc.edu

Key words: radiation therapy, signaling pathways, cell cycle checkpoint, DNA repair, cell survival
6. DNA repair pathways

7. Conclusion

\section{Introduction}

As a staple approach for cancer treatment, radiation therapy plays a critical role in local disease control. When combined with chemotherapy (i.e., chemoradiation), radiation provides additional benefits, which give better disease control and significantly improve cancer patient survival (1-3). However, radioresistance and the presence of residual disease after radiation therapy remain major problems that result in the loss of the therapy effectiveness (4-7). Currently, there is no clinical approach available either for predicting the benefit of radiation therapy for individual cancer patients or for radiosensitization of cancer cells. Thus, an improved understanding of the mechanisms that promote cancer cell survival after radiation could allow pharmacological strategies to be developed to improve the efficacy of radiation therapy.

Radiation exposure induces numerous cellular signaling pathways, which can lead to cellular responses including apoptosis, cellular senescence and cell cycle checkpoint activation/ DNA repair (8). Among the radiation-induced pro-survival signaling pathways, some are involved in inducing cell cycle arrest and promoting DNA repair, while others are engaged in suppressing apoptosis induction $(9,10)$. These pathways act synergistically to protect cancer cells from the cytotoxic effects of radiation, ultimately leading to the development of radioresistance. This review summarizes the signaling pathways that positively contribute to cancer cell survival in response to ionizing radiation.

\section{HER (also called ERBB or EGFR) signaling}

The HER family of receptor tyrosine kinases (RTKs) consists of HER1, HER2, HER3 and HER4, which localize on the cell membrane (11). HER RTKs share a similar protein structure that contains an extracellular region (ligand binding and dimerization domains), a transmembrane region and an intracellular region (protein tyrosine kinase domain and phosphorylation regulatory tail) (12). Among HER receptors, HER2 has no known ligand and HER3 possesses very low kinase activity (12). Binding of ligands to the ligand binding domain of HER1, 
HER3 and HER4 results in homo- or hetero-dimerization of the receptors followed by trans-phosphorylation of several tyrosines in the c-terminal regulatory tail of the receptor (12). The phosphorylated tyrosines form docking sites for downstream adaptors and signal transducers, activating downstream signaling pathways including PI3K/AKT, RAS/RAF/MEK/ ERK, phospholipase $\mathrm{C}-\gamma /$ protein kinase $\mathrm{C}$ and JAK/STAT pathways $(13,14)$. Among those pathways, PI3K/AKT and RAS/RAF/MEK/ERK cascades have been shown to play important roles in cell survival after radiation (Fig. 1) (15).

An increase in HER1 phosphorylation, indicative of HER activation, following ionizing radiation has been reported previously (16-18). Our most recent study in human breast cancer cells demonstrates that ionizing radiation results in an increase in phosphorylation of not only HER1, but also HER2, HER3 and HER4 (19). Although the mechanisms responsible for this phosphorylation of HER receptors has not yet been determined, previous studies have shown that receptor protein tyrosine phosphatases (PTPs), which suppress HER RTK phosphorylation, can effectively be inhibited by reactive oxygen/nitrogen species (ROS/RNS) through oxidation (20). Previous studies have also demonstrated that radiation induces ROS/RNS production via a mitochondria-dependent mechanism (21). Thus, the ROS/RNS production in response to radiation could lead to the inhibition of PTPs, resulting in the activation of HER RTKs. Future studies will be needed to examine this possibility for the activation of HER RTKs following radiation.

Inhibition of HER RTKs has been shown to increase the radiosensitivity of cancer cells. Inhibition of HER RTKs by HER pan-inhibitor CI-1033 notably enhances the radiosensitivity of human colon carcinoma cells both in vitro and in vivo (22), while HER1 inhibition by gefitinib and HER2 inhibition by herceptin, respectively, radiosensitizes EGFR amplified glioma cells and breast cancer cells $(23,24)$. Generally, the pro-survival function of HER receptors involves at least two possible mechanisms. The first mechanism is based on the capability of HER receptors to activate AKT and ERK1/2 signaling, which play critical roles in suppressing apoptosis (15). Another possible mechanism for the pro-survival function of HER receptors is through their regulation of the cell cycle checkpoint response and DNA repair. In our recent study, we found that HER2 activation following radiation is necessary for the activation of the G2/M cell cycle checkpoint response (19). In addition, HER1 has been reported to promote the activation of DNA-dependent protein kinase (DNA-PK), which plays an essential role in the NHEJ-mediated repair of DNA double-strand breaks (DSBs) $(25,26)$.

\section{Extracellular signal-regulated kinase (ERK1/2) pathway}

In a wide variety of cell types, ionizing radiation induces rapid activation of MAPK family members, including ERK1/2, JNK and p38 (27,28). Among those, radiation-induced ERK1/2 signaling activation has been shown to play an important role in promoting cell survival in response to radiation (29-31).

Following radiation, ERK1/2 is activated through dual tyrosine and threonine phosphorylation by MEK1/2 and the activation, in turn, leads to the phosphorylation/activation of over 160 substrates (32). Some of these substrates are tran- scription factors that regulate the expression of genes encoding for anti-apoptotic proteins $(27,32)$. The best characterized antiapoptotic transcription factors targeted by ERK1/2 signaling are the cyclic AMP-responsive element binding protein (CREB) and CAAT/enhancer binding protein $\beta(\mathrm{C} / \mathrm{EBP}-\beta)$. In response to radiation, ERK1/2 phosphorylates/activates p $90^{\text {rsk }}$ kinase, which in turn activates $\mathrm{CREB}$ and $\mathrm{C} / \mathrm{EBP}-\beta$, thereby inducing the expression of a number of anti-apoptotic proteins including Bcl-xL, Mcl-1 and c-FLIPs (33-35). In addition, ERK1/2 can directly phosphorylate and inhibit a number of pro-apoptotic proteins, including Bad, Bim and caspase 9 (36-39). Thus, by increasing the expression/activity of anti-apoptotic proteins and inhibiting the activity of pro-apoptotic proteins, the net effect of the radiation-induced ERK1/2 signaling activation is the suppression of apoptosis in irradiated cells.

Studies from our group and others have demonstrated that ERK1/2 signaling activation after radiation is essential for activation of the G2/M cell cycle checkpoint in response to radiation (29,31,40-42). Radiation-induced ERK1/2 signaling is required for the activation of key regulators of the G2 checkpoint, most notably ATR and BRCA1 $(31,42)$. ERK1/2 signaling also plays an important role in promoting DNA repair. Radiation-induced ERK1/2 signaling has been associated with the transcriptional upregulation of genes involved in DNA repair, such as ERCC1, XRCC1 and XPC $(43,44)$. Furthermore, ERK1/2 signaling can activate DNA-PK, which plays a critical role in NHEJ-mediated DSB repair, and PARP-1, which recognizes single-stranded DNA breaks (SSBs) on the damaged DNA (44-47). Also, ERK1/2 signaling functions as a positive regulator of ataxia telangiectasia mutated (ATM)-dependent homologous recombination (HR) DSB repair (48). Thus, by promoting G2/M cell cycle checkpoint activation and increasing DNA repair, ERK1/2 signaling positively regulates cancer cell survival following radiation. Consistent with these observations, an increasing number of studies demonstrate that constitutive activation of Ras results in an increase in the radioresistance of cancer cells, whereas inhibition of MEK or ERK leads to the radiosensitization of cancer cells $(29,40,41,49)$.

While the exact mechanisms responsible for the activation of ERK1/2 signaling by radiation has not yet been clearly elucidated, several signaling mechanisms have been proposed to be involved in this activation. As demonstrated by us and others, the rapid activation of HER family receptors following ionizing radiation contributes to ERK1/2 signaling activation in cancer cells of the breast and lung (17). Furthermore, this role of HER receptors involves Ras GTPase. An activation of Ras in response to HER receptor activation (mainly HER1 and HER2) has been demonstrated and ectopic expression of Ras-N17 dominant negative mutant abolishes the ERK1/2 activation by radiation $(50,51)$. Via recruitment of Grb-2 to the activated HER receptors, Grb-2 becomes activated and forms a complex with SOS protein, which triggers the activation of Ras/Raf/MEK/ERK signaling (Fig. 1) (50,51). Furthermore, the activated Ras can induce HER1-ligand production, which, through an autocrine feedback loop, further activates HER1 and then Ras/Raf/MEK/ERK signaling $(52,53)$. Another mechanism implicated in radiation-induced ERK1/2 activation involves the tumor suppressor BRCA1. Studies from our laboratory show that decreasing BRCA1 expression in breast 


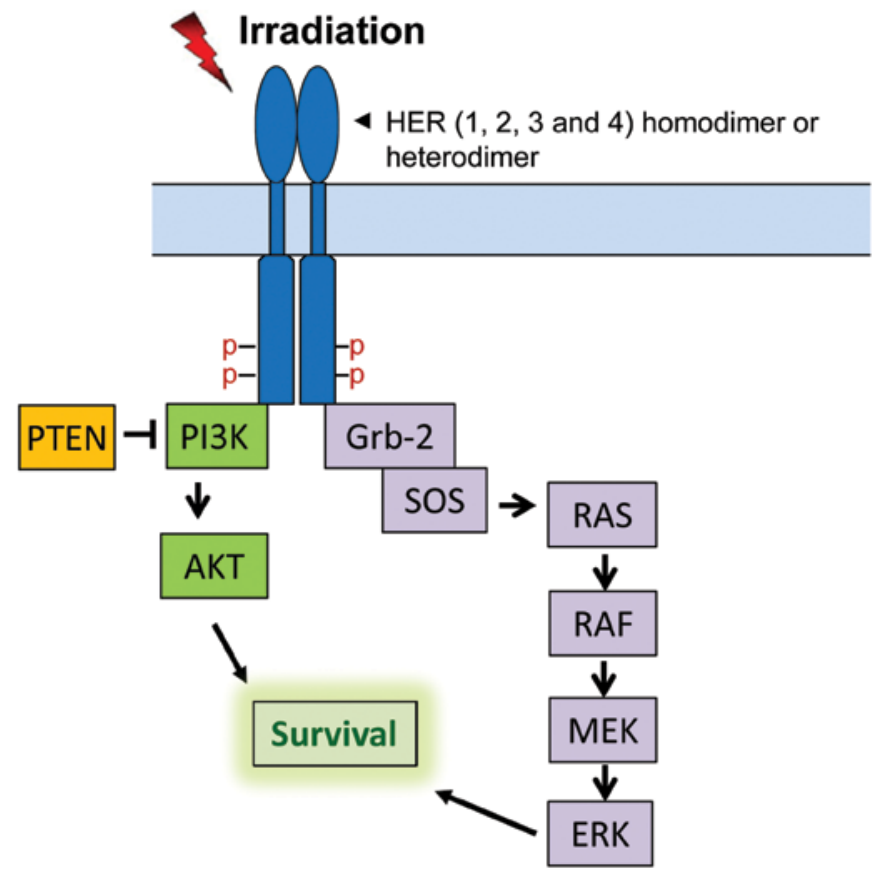

Figure 1. Radiation induces activation of HER receptors, which, in turn, leads to the activation of PI3K/AKT and RAS/RAF/MEK/ERK signaling pathways that promote cell survival.

cancer cells using shRNA markedly diminishes the activation of ERK1/2 signaling after radiation (42). Conversely, inhibition of ERK1/2 signaling using pharmacological inhibitors or siRNA also results in the destabilization of BRCA1 protein in irradiated breast cancer cells (42). These results suggest a positive feedback loop involving ERK1/2 and BRCA1 in response to ionizing radiation. Lastly, the DNA damage sensor ATM has also been implicated in radiation-induced ERK1/2 activation (48). ERK1/2 activation following radiation has been shown to require ATM, as ATM inhibition partially blocks the radiation-induced ERK1/2 activation (48). Conversely, inhibition of ERK1/2 signaling can also attenuate radiation-induced ATM phosphorylation, as well as the recruitment of ATM to DNA damage foci (48). These studies suggest another positive feedback loop in the radiation response, this time involving ATM and ERK1/2. Collectively, these studies indicate that the activation of ERK1/2 signaling in response to radiation is regulated by multiple inter-regulated signaling pathways.

\section{AKT signaling pathway}

The AKT signaling pathway plays a vital role in cell survival. Aberrant activation of this signaling cascade has been detected in various types of malignancies and is associated with tumorigenesis (54). AKT functions as a pro-survival factor by inhibiting apoptotic signal cascades and activating pro-survival signaling pathways (Fig. 2). Upon activation, AKT phosphorylates and inhibits a number of pro-apoptotic Bcl-2 family members, including Bad, Bax and Bim (55-57). Furthermore, through direct inhibition and exclusion of proapoptotic transcription factor FOXO3a (Forkhead box O3), AKT also suppresses the expressions of the pro-apoptotic factors Bim and Noxa (58-61).

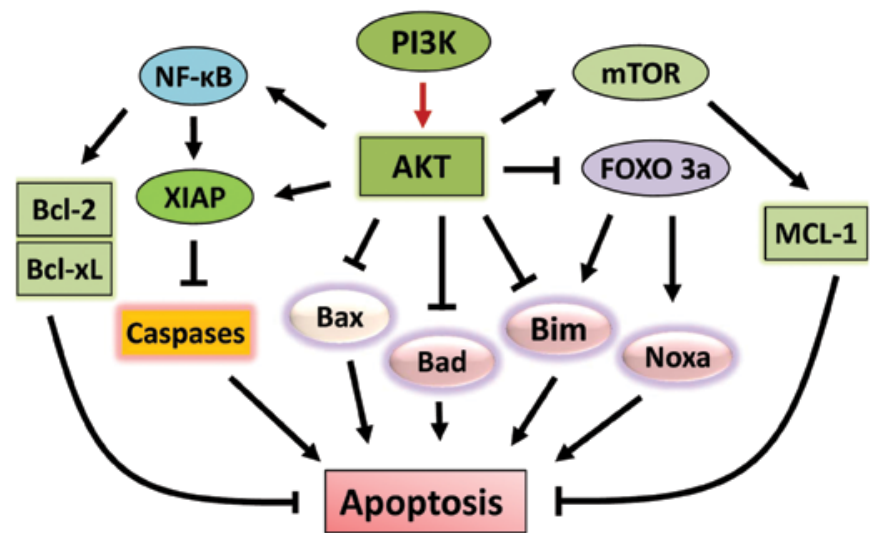

Figure 2. PI3K/AKT mediated signaling promotes cell survival. i) Activation of PI3K by radiation leads to the phosphorylation/activation of AKT; ii) AKT phosphorylates and inhibits pro-apoptotic proteins Bad, Bax, Bim and Noxa; iii) AKT phosphorylates and activates pro-survival transcription factor NF- $\mathrm{BB}$, leading to the upregulation of pro-survival genes $B C L-2$ and $B C L-X L$; iv) AKT phosphorylates pro-survival protein XIAP, which binds and inhibits caspase 3/7/9, which are required for apoptosis induction; v) AKT phosphorylates/activates mTOR kinase, which phosphorylates/activates antiapoptotic protein Mcl-1; vi) FOXO3a upregulates the gene expression of pro-apoptotic proteins Bim and Noxa. Phosphorylation of FOXO3a by AKT results in inhibition and nuclei exclusion of the protein.

AKT also positively regulates anti-apoptotic pathways (Fig. 2). AKT induces activation of $N F-\kappa B$ transcription factor, which promotes the transcription of a wide range of anti-apoptotic genes, in particular $B C L-2$ and $B C L-X L$ (62). Furthermore, AKT phosphorylates/activates pro-survival protein XIAP (X-linked inhibitor of apoptosis protein), resulting in an increase of binding of XIAP to caspases 3, 7 and 9 and inhibition of these caspases, the activities of which are essential for apoptosis induction (63). Another key pro-survival pathway targeted by AKT is the mTOR signaling pathway. AKT phosphorylates and activates mTOR kinase, leading to the phosphorylation/activation of anti-apoptotic protein Mcl-1 (64,65). Furthermore, AKT negatively regulates hypoxia-induced apoptosis. Following radiation therapy, hypoxia is often induced in tissues by radiation and can lead to apoptosis in the injured tissue $(66,67)$. The hypoxia-induced apoptosis requires glycogen synthase kinase (GSK) to activate the mitochondria-dependent death signaling pathway $(67,68)$. However, AKT activation following radiation can inhibits the activity of GSK through phosphorylation, resulting in an activation of glycolysis and glucose transport that suppresses apoptosis induction (69). Lastly, AKT is directly involved in the activation of the catalytic subunit of DNA-PK after radiation, promoting NHEJ-mediated DSB repair that increases cell survival (70). These studies establish a pro-survival role for AKT mediated signaling pathways in the response of cancer cells to radiation.

Activation of the PI3K/AKT signaling pathway in response to ionizing radiation has been widely observed (15). A likely mechanism for this activation involves HER RTKs. Upon activation of HER RTKs, the phosphorylated tyrosines in the carboxyl-terminal regulatory tail of HER3 can form six docking sites for recruitment of the p85 adaptor subunit of phosphatidylinositol 3-kinase (PI3K) (71). 


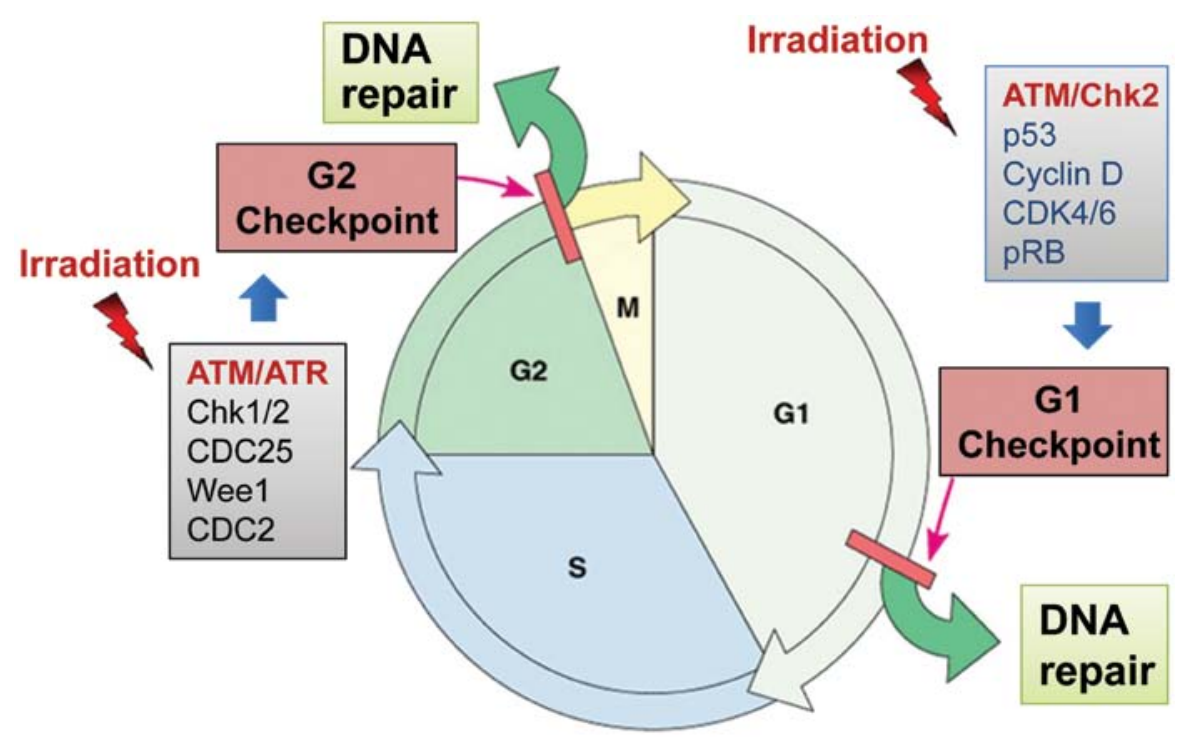

Figure 3. Irradiation induces G1 and G2 cell cycle checkpoint activation and DNA repair. Most cancer cells are defective in G1 checkpoint, commonly due to the mutations/alterations of the key regulators of the G1 checkpoint (in blue), but contain a functional G2 checkpoint.

Subsequently, the p110 catalytic subunit of PI3K phosphorylates phosphatidylinositol-4,5-biphosphate (PIP2) to generate phosphatidylinositol $(3,4,5)$-triphosphate (PIP3), which then leads to the membrane recruitment and activation of proteins that contain a phospholipid-binding $(\mathrm{PH})$ domain, such as phosphoinositide-dependent kinase (PDK) 1 (72). The activated PDK1 phosphorylates AKT-Thr308 and leads to the initial AKT activation (72). The full-activation of AKT requires further phosphorylation of its Ser473 by PDK2 (72). Furthermore, mutant K-Ras also positively contributes to the activation of PI3K-AKT signaling in response to radiation, which is through its activation of autocrine production of EGFR ligands (73,74).

The pro-survival function of PI3K/AKT signaling is expected to positively contribute to the radioresistance of tumor cells. Indeed, an increasing number of studies indicate that inhibition of PI3K/AKT signaling by either pharmacological inhibitors or genetic approaches leads to an enhancement of radiosensitivity of cancer cells both in vitro and in vivo (75-77). Furthermore, the increase in radiosensitivity by PI3K/AKT inhibition involves both the diminution of DNA repair and an enhancement of apoptosis induction $(70,75,76,78,79)$. On the other hand, in some cell-based models, inhibition of PI3K/AKT has been shown to have little effect on radiosensitivity (29,80-83), suggesting an involvement of $\mathrm{PI} 3 \mathrm{~K} / \mathrm{AKT}$-independent mechanisms in the regulation of radiosensitivity.

\section{Cell cycle checkpoint signaling}

In response to DNA damage, cell cycle checkpoints often become activated to block cell cycle progression, allowing time for cells to repair the damage (84). Depending on the phase of the cell cycle at which the damage is sensed, the cells can be blocked at the G1/S or G2/M border of the cell cycle (Fig. 3) (84). If the damage is irreversible or the cell cycle checkpoint is dysfunctional, apoptosis may be triggered

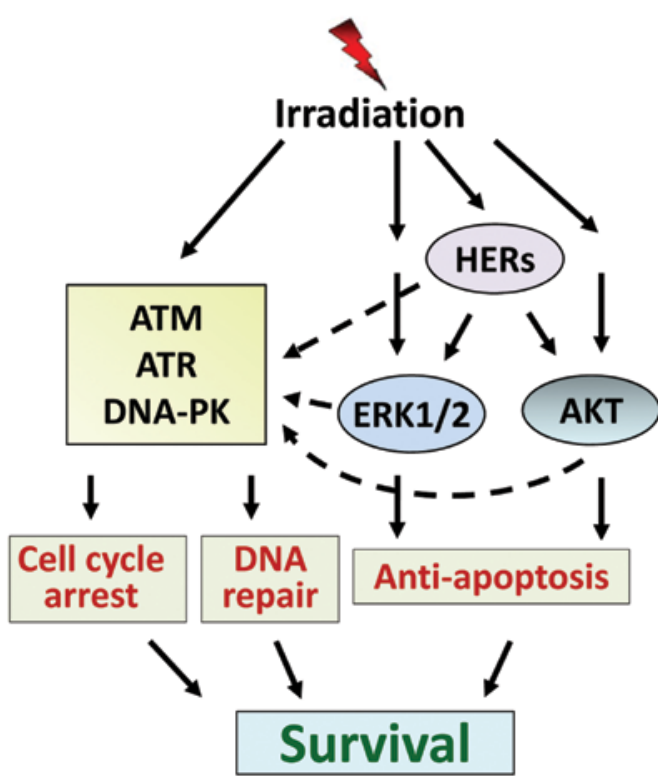

Figure 4. Overview of radiation-induced signaling pathways that promote cell survival. Activation of ATM, ATR and DNA-PK signaling by radiation leads to cell cycle arrest and DNA repair. Activation of HER, ERK1/2 and AKT signaling pathways by radiation suppresses apoptosis induction. HER, ERK1/2 and AKT signaling activation following radiation positively regulate cell cycle checkpoint response and DNA repair.

to eliminate the injured cells (84). Thus, properly functioning cell cycle checkpoints promote cell survival by counteracting the cytotoxicity of DNA damage.

The G1/S transition is controlled by the activity of Cdk4/6 kinases coupled with Cyclin D, the activities of which are predominantly regulated by the p53/p21 pathway (80). The $\mathrm{G} 2 / \mathrm{M}$ border is tightly controlled by the $\mathrm{Cdc} 2 / \mathrm{Cyclin} \mathrm{B}$ complex, whose activity is required for the $\mathrm{G} 2 / \mathrm{M}$ transition of the cell cycle (85). The G1 checkpoint is defective in most cancer cells, commonly due to mutations/alterations of key regulators of the G1 checkpoint (p53, Cyclin D, etc.) (80), 
whereas activation of the G2 checkpoint is rarely impaired in cancer cells, as this checkpoint operates primarily via a p53-independent mechanism (Fig. 3) (86). In fact, in cancer cells lacking a functional G1 checkpoint, abrogation of the G2 checkpoint often sensitizes the cells to radiation (87).

Previous studies identified Cdc2-Y15 as a vital site involved in G2 checkpoint control in response to radiation (88). Cdc2-Y15 is phosphorylated in response to radiation exposure and this phosphorylation is maintained during radiationinduced G2/M arrest (88-90). Cdc2-Y15 is phosphorylated by the Wee1 and Myt1 kinases $(91,92)$ and dephosphorylated by the Cdc25 dual-specificity phosphatases (93).

ATM- and ATR-mediated signaling pathways play essential roles in the radiation-induced cell cycle checkpoint responses (84). In response to radiation-induced DNA-damage, ATM and ATR kinases are rapidly activated, which, in turn, activate their respective downstream targets, including p53 as well as the Chk1 and Chk2 kinases (Fig. 3) (84). Activation of Chk1 and Chk2 results in phosphorylation of $\mathrm{Cdc} 25$, leading to the subcellular sequestration, degradation and/or inhibition of the Cdc25 that normally activates Cdc2/Cyclin B at the G2/M boundary (94). Chk2 can also phosphorylate p53-Ser20 to induce stabilization of the $\mathrm{p} 53$ protein following radiation (84). Activation of p53 by ATM, ATR and Chk2 kinases leads to the induction of $\mathrm{p} 21$ protein, which can directly inhibit the activity of the Cdc2/Cyclin B complex (84).

In summary, radiation-induced cell cycle checkpoint signaling pathways promote cell cycle arrest, which, in turn, contributes positively to cell survival in response to radiation.

\section{DNA repair pathways}

The cytotoxicity caused by ionizing radiation is mainly the result of DNA damage. Radiation induces several forms of DNA damage, which include SSBs, DSBs, sugar and base modifications and DNA-protein crosslinks $(95,96)$. Among these, DSBs are not only a dominant form of damage caused by ionizing radiation $(97,98)$, but also is the most deadly form of DNA damage, as unrepaired DSBs can lead to lethality of cells $(97,99)$.

In response to ionizing radiation, the activation of the phosphoinositide 3-kinase-related kinases (PIKKs), including ATM, ATR and DNA-PK, transduces and amplifies the DNA-damage signal, triggering the assembly of DNA repair apparatuses at the damaged sites and initiating DNA repair (10). A DSB is repaired by one of two competing mechanisms: non-homologous end joining repair (NHEJ) and homologous recombination (HR) (10), with both mechanisms regulated by PIKKs. With no sequence homology required, NHEJ rejoins the free ends in a process that commonly produces errors at the point of junction (100). Each of the two ends is recognized by the Ku70/Ku80 heterodimer, which then recruits DNA-PK (100). Once formed, these complexes bring the ends together for further processing and ligation by DNA ligase IV (100). In contrast to NHEJ, HR repairs DSBs accurately and with very high fidelity (100). This process operates during the S and G2 phases and repairs DSBs taking advantage of sequence information present in the intact sister chromatid (100). Radiation also creates SSBs, mainly through base oxidation driven by ROS/RNS (98). The repair of this type of damage uses base excision repair, which removes the damaged base using a DNA glycosylase and AP endonuclease and then fills up the nick through the actions of DNA polymerases and DNA ligase (101). Consequently, successful DNA repairs promote cell survival in response to radiation, whereas a failure to repair the DNA damage enhances the cytotoxic effect of radiation, leading to lethality of the cells.

\section{Conclusion}

As a standard of care, radiation therapy plays an important role in cancer therapy. However, radiation resistance remains a major obstacle that limits the efficacy of radiation therapy for cancer treatment. In order to improve the efficacy of radiation therapy, it is necessary that we fully understand the signaling network that drives cancer cells to overcome radiation-induced cytotoxicity, leading to survival. As discussed above, the lethal cytotoxicity caused by ionizing radiation is mainly the result of DSBs. However, radiation also simultaneously induces multiple signaling pathways that can protect cells from the cytotoxic effect of radiation. Among these, signalings mediated by HER receptors, ERK1/2 and AKT prevent the irradiated cells from undergoing apoptosis induction, while signalings mediated by ATM, ATR and DNA-PK drive cells into cycle arrest and initiate DNA repair. In addition, HER ERK1/2 and AKT signaling also positively regulate the cell cycle checkpoint response and DNA repair machinery. Consequently, these signaling pathways act conjointly to rescue the cells from radiation-induced injury and promote survival (Fig. 4). To overcome radiation therapy resistance, future research should focus on the development of pharmacological approaches to block the activation of these pro-survival signaling pathways in irradiated cells.

\section{Acknowledgements}

This study was supported by a Nebraska DHHS-LB506 grant 2010-40 to Y.Y. and NCI SPORE grant (P50 CA127297) to M.M.O.

\section{References}

1. Pignon JP, le Maitre A, Maillard E and Bourhis J: Meta-analysis of chemotherapy in head and neck cancer (MACH-NC): an update on 93 randomised trials and 17,346 patients. Radiother Oncol 92: 4-14, 2009.

2. Ramnath N, Dilling TJ, Harris LJ, et al: Treatment of stage III non-small cell lung cancer: diagnosis and management of lung cancer, 3rd ed: American College of Chest Physicians evidencebased clinical practice guidelines. Chest 143: eS314-eS340, 2013.

3. Wilkinson-Ryan I, Binder PS, Pourabolghasem S, et al: Concomitant chemotherapy and radiation for the treatment of advanced-stage endometrial cancer. Gynecol Oncol 134: 24-28, 2014.

4. Johnstone RW, Ruefli AA and Lowe SW: Apoptosis: a link between cancer genetics and chemotherapy. Cell 108: 153-164, 2002.

5. Milas L, Raju U, Liao Z and Ajani J: Targeting molecular determinants of tumor chemo-radioresistance. Semin Oncol 32: S78-S81, 2005.

6. Bernier J: Current state-of-the-art for concurrent chemoradiation. Semin Radiat Oncol 19: 3-10, 2009.

7. Ghiam AF, Spayne J and Lee J: Current challenges and future perspectives of radiotherapy for locally advanced breast cancer. Curr Opin Support Palliat Care 8: 46-52, 2014. 
8. Gewirtz DA: Growth arrest and cell death in the breast tumor cell in response to ionizing radiation and chemotherapeutic agents which induce DNA damage. Breast Cancer Res Treat 62: 223-235, 2000

9. Hawkins AJ, Golding SE, Khalil A and Valerie K: DNA doublestrand break-induced pro-survival signaling. Radiother Oncol 101: 13-17, 2011.

10. Raleigh DR and Haas-Kogan DA: Molecular targets and mechanisms of radiosensitization using DNA damage response pathways. Future Oncol 9: 219-233, 2013.

11. Navolanic PM, Steelman LS and McCubrey JA: EGFR family signaling and its association with breast cancer development and resistance to chemotherapy (Review). Int J Oncol 22: 237-252, 2003.

12. Linggi B and Carpenter G: ErbB receptors: new insights on mechanisms and biology. Trends Cell Biol 16: 649-656, 2006.

13. Arteaga Carlos L and Engelman Jeffrey A: ERBB receptors: from oncogene discovery to basic science to mechanism-based cancer therapeutics. Cancer Cell 25: 282-303, 2014.

14. Rexer BN and Arteaga CL: Intrinsic and acquired resistance to HER2-targeted therapies in HER2 gene-amplified breast cancer: mechanisms and clinical implications. Crit Rev Oncog 17: 1-16, 2012.

15. Valerie K, Yacoub A, Hagan MP, et al: Radiation-induced cell signaling: inside-out and outside-in. Mol Cancer Ther 6: 789-801, 2007.

16. Goldkorn T, Balaban N, Shannon M and Matsukuma K: EGF receptor phosphorylation is affected by ionizing radiation. Biochim Biophys Acta 1358: 289-299, 1997.

17. Lee H-C, An S, Lee H, et al: Activation of epidermal growth factor receptor and its downstream signaling pathway by nitric oxide in response to ionizing radiation. Mol Cancer Res 6 : 996-1002, 2008

18. Kiyozuka M, Akimoto T, Fukutome M, Motegi A and Mitsuhashi N: Radiation-induced dimer formation of EGFR: implications for the radiosensitizing effect of cetuximab. Anticancer Res 33: 4337-4346, 2013.

19. Yan Y, Hein AL, Greer PM, et al: A novel function of HER2/Neu in the activation of $\mathrm{G} 2 / \mathrm{M}$ checkpoint in response to [gamma] irradiation. Oncogene: June 9, 2014 (Epub ahead of print). doi: 10.1038/onc.2014.167.

20. Meng TC, Fukada T and Tonks NK: Reversible oxidation and inactivation of protein tyrosine phosphatases in vivo. Mol Cell 9: 387-399, 2002

21. Leach JK, Van Tuyle G, Lin PS, Schmidt-Ullrich R and Mikkelsen RB: Ionizing radiation-induced, mitochondriadependent generation of reactive oxygen/nitrogen. Cancer Res 61: 3894-3901, 2001.

22. Nyati MK, Maheshwari D, Hanasoge S, et al: Radiosensitization by Pan ErbB Inhibitor CI-1033 in vitro and in vivo. Clin Cancer Res 10: 691-700, 2004.

23. Liang K, Lu Y, Jin W, Ang KK, Milas L and Fan Z: Sensitization of breast cancer cells to radiation by trastuzumab. Mol Cancer Ther 2: 1113-1120, 2003.

24. Geoerger B, Gaspar N, Opolon P, et al: EGFR tyrosine kinase inhibition radiosensitizes and induces apoptosis in malignant glioma and childhood ependymoma xenografts. Int J Cancer 123 : 209-216, 2008.

25. Dittmann K, Mayer C, Fehrenbacher B, et al: Radiation-induced epidermal growth factor receptor nuclear import is linked to activation of DNA-dependent protein kinase. J Biol Chem 280: 31182-31189, 2005

26. Dittmann K, Mayer C and Rodemann HP: Inhibition of radiationinduced EGFR nuclear import by C225 (Cetuximab) suppresses DNA-PK activity. Radiother Oncol 76: 157-161, 2005

27. Dent P, Yacoub A, Fisher PB, Hagan MP and Grant S: MAPK pathways in radiation responses. Oncogene 22: 5885-5896, 2003

28. Cui W, Yazlovitskaya EM, Mayo MS, Pelling JC and Persons DL: Cisplatin-induced response of c-jun N-terminal kinase 1 and extracellular signal-regulated protein kinases 1 and 2 in a series of cisplatin-resistant ovarian carcinoma cell lines. Mol Carcinog 29: $219-228,2000$

29. Abbott DW and Holt JT: Mitogen-activated protein kinase kinase 2 activation is essential for progression through the G2/M checkpoint arrest in cells exposed to ionizing radiation. $\mathrm{J}$ Biol Chem 274: 2732-2742, 1999.

30. Tang D, Wu D, Hirao A, et al: ERK activation mediates cell cycle arrest and apoptosis after DNA damage independently of p53. J Biol Chem 277: 12710-12717, 2002.
31. Yan Y, Black CP and Cowan KH: Irradiation-induced G2/M checkpoint response requires ERK1/2 activation. Oncogene 26: 4689-4698, 2007.

32. Munshi A and Ramesh R: Mitogen-activated protein kinases and their role in radiation response. Genes Cancer 4: 401-408, 2013.

33. Boucher MJ, Morisset J, Vachon PH, Reed JC, Laine J and Rivard N: MEK/ERK signaling pathway regulates the expression of Bcl-2, Bcl-X(L), and Mcl-1 and promotes survival of human pancreatic cancer cells. J Cell Biochem 79: 355-369, 2000.

34. Aoudjit F and Vuori K: Matrix attachment regulates Fas-induced apoptosis in endothelial cells: a role for c-flip and implications for anoikis. J Cell Biol 152: 633-643, 2001.

35. Jost M, Huggett TM, Kari C, Boise LH and Rodeck U: Epidermal growth factor receptor-dependent control of keratinocyte survival and Bcl-xL expression through a MEK-dependent pathway. J Biol Chem 276: 6320-6326, 2001.

36. Bonni A, Brunet A, West AE, Datta SR, Takasu MA and Greenberg ME: Cell survival promoted by the Ras-MAPK signaling pathway by transcription-dependent and -independent mechanisms. Science 286: 1358-1362, 1999.

37. Clark CJ, McDade DM, O'Shaughnessy CT and Morris BJ: Contrasting roles of neuronal Msk1 and Rsk2 in Bad phosphorylation and feedback regulation of Erk signalling. J Neurochem 102: 1024-1034, 2007

38. Ewings KE, Hadfield-Moorhouse K, Wiggins CM, et al: ERK1/2-dependent phosphorylation of BimEL promotes its rapid dissociation from Mcl-1 and Bcl-xL. EMBO J 26: 2856-2867, 2007.

39. Allan LA, Morrice N, Brady S, Magee G, Pathak S and Clarke PR: Inhibition of caspase-9 through phosphorylation at Thr 125 by ERK MAPK. Nat Cell Biol 5: 647-654, 2003.

40. Tamamoto T, Ohnishi K, Takahashi A, et al: Correlation between gamma-ray-induced G2 arrest and radioresistance in two human cancer cells. Int J Radiat Oncol Biol Phys 44: 905-909, 1999.

41. Fritz G, Brachetti C and Kaina B: Lovastatin causes sensitization of HeLa cells to ionizing radiation-induced apoptosis by the abrogation of G2 blockage. Int J Radiat Biol 79: 601-610, 2003.

42. Yan Y, Black CP, Cao PT, et al: Gamma-irradiation-induced DNA damage checkpoint activation involves feedback regulation between extracellular signal-regulated kinase $1 / 2$ and BRCA1. Cancer Res 68: 5113-5121, 2008.

43. Yacoub A, McKinstry R, Hinman D, Chung T, Dent P and Hagan MP: Epidermal growth factor and ionizing radiation up-regulate the DNA repair genes XRCC1 and ERCC1 in DU145 and LNCaP prostate carcinoma through MAPK signaling. Radiat Res 159: 439-452, 2003.

44. Golding SE, Morgan RN, Adams BR, Hawkins AJ, Povirk LF and Valerie K: Pro-survival AKT and ERK signaling from EGFR and mutant EGFRvIII enhances DNA double-strand break repair in human glioma cells. Cancer Biol Ther 8: 730-738, 2009.

45. Wei F, Yan J, Tang D, et al: Inhibition of ERK activation enhances the repair of double-stranded breaks via non-homologous end joining by increasing DNA-PKcs activation. Biochim Biophys Acta 1833: 90-100, 2013.

46. Cohen-Armon M: PARP-1 activation in the ERK signaling pathway. Trends Pharmacol Sci 28: 556-560, 2007.

47. Cohen-Armon M, Visochek L, Rozensal D, et al: DNA-independent PARP-1 activation by phosphorylated ERK2 increases Elk1 activity: a link to histone acetylation. Mol Cell 25: 297-308, 2007.

48. Golding SE, Rosenberg E, Neill S, Dent P, Povirk LF and Valerie K: Extracellular signal-related kinase positively regulates ataxia telangiectasia mutated, homologous recombination repair, and the DNA damage response. Cancer Res 67: 1046-1053, 2007.

49. Yan Y, Greer PM, Cao PT, Kolb RH and Cowan KH: RACl GTPase plays an important role in gamma-irradiation induced G2/M checkpoint activation. Breast Cancer Res 14: R60, 2012.

50. Sasaoka T, Langlois WJ, Leitner JW, Draznin B and Olefsky JM: The signaling pathway coupling epidermal growth factor receptors to activation of p21ras. J Biol Chem 269: 32621-32625, 1994.

51. Janes PW, Daly RJ, deFazio A and Sutherland RL: Activation of the Ras signalling pathway in human breast cancer cells overexpressing erbB-2. Oncogene 9: 3601-3608, 1994.

52. Dent P, Reardon DB, Park JS, et al: Radiation-induced release of transforming growth factor alpha activates the epidermal growth factor receptor and mitogen-activated protein kinase pathway in carcinoma cells, leading to increased proliferation and protection from radiation-induced cell death. Mol Biol Cell 10: 2493-2506, 1999. 
53. Hagan M, Wang L, Hanley JR, Park JS and Dent P: Ionizing radiation-induced mitogen-activated protein (MAP) kinase activation in DU145 prostate carcinoma cells: MAP kinase inhibition enhances radiation-induced cell killing and G2/M-phase arrest. Radiat Res 153: 371-383, 2000.

54. Polivka J Jr and Janku F: Molecular targets for cancer therapy in the PI3K/AKT/mTOR pathway. Pharmacol Ther 142: 164-175, 2014.

55. Yamaguchi $\mathrm{H}$ and Wang HG: The protein kinase PKB/Akt regulates cell survival and apoptosis by inhibiting Bax conformational change. Oncogene 20: 7779-7786, 2001.

56. Gardai SJ, Hildeman DA, Frankel SK, et al: Phosphorylation of Bax Ser184 by Akt regulates its activity and apoptosis in neutrophils. J Biol Chem 279: 21085-21095, 2004

57. Qi XJ, Wildey GM and Howe PH: Evidence that Ser87 of BimEL is phosphorylated by Akt and regulates BimEL apoptotic function. J Biol Chem 281: 813-823, 2006.

58. Engström M, Karlsson R and Jönsson J-I: Inactivation of the forkhead transcription factor FoxO3 is essential for PKB-mediated survival of hematopoietic progenitor cells by kit ligand. Exp Hematol 31: 316-323, 2003.

59. Yang JY, Xia W and Hu MC: Ionizing radiation activates expression of FOXO3a, Fas ligand, and Bim, and induces cell apoptosis. Int J Oncol 29: 643-648, 2006

60. Obexer P, Geiger K, Ambros PF, Meister B and Ausserlechner MJ: FKHRL1-mediated expression of Noxa and Bim induces apoptosis via the mitochondria in neuroblastoma cells. Cell Death Differ 14: 534-547, 2007.

61. Jang S-W, Yang S-J, Srinivasan S and Ye K: Akt phosphorylates MstI and prevents its proteolytic activation, blocking FOXO3 phosphorylation and nuclear translocation. J Biol Chem 282: 30836-30844, 2007

62. Ozes ON, Mayo LD, Gustin JA, Pfeffer SR, Pfeffer LM and Donner DB: NF-kappaB activation by tumour necrosis factor requires the Akt serine-threonine kinase. Nature 401: 82-85, 1999.

63. Dan HC, Sun M, Kaneko S, et al: Akt phosphorylation and stabilization of X-linked inhibitor of apoptosis protein (XIAP). J Biol Chem 279: 5405-5412, 2004.

64. Shaw RJ and Cantley LC: Ras, PI(3)K and mTOR signalling controls tumour cell growth. Nature 441: 424-430, 2006

65. Fumarola C, Bonelli MA, Petronini PG and Alfieri RR: Targeting PI3K/AKT/mTOR pathway in non small cell lung cancer. Biochem Pharmacol 90: 197-207, 2014

66. Fleckenstein K, Zgonjanin L, Chen L, et al: Temporal onset of hypoxia and oxidative stress after pulmonary irradiation. Int J Radiat Oncol Biol Phys 68: 196-204, 2007.

67. Sendoel A and Hengartner MO: Apoptotic cell death under hypoxia. Physiology 29: 168-176, 2014.

68. King TD, Bijur GN and Jope RS: Caspase- 3 activation induced by inhibition of mitochondrial complex I is facilitated by glycogen synthase kinase- $3 \beta$ and attenuated by lithium. Brain Res 919: 106-114, 2001

69. Loberg RD, Vesely E and Brosius FC: Enhanced glycogen synthase kinase-3 $\beta$ activity mediates hypoxia-induced apoptosis of vascular smooth muscle cells and is prevented by glucose transport and metabolism. J Biol Chem 277: 41667-41673, 2002.

70. Toulany M, Kehlbach R, Florczak U, et al: Targeting of AKT1 enhances radiation toxicity of human tumor cells by inhibiting DNA-PKcs-dependent DNA double-strand break repair. Mol Cancer Ther 7: 1772-1781, 2008.

71. Soltoff SP, Carraway KL III, Prigent SA, Gullick WG and Cantley LC: ErbB3 is involved in activation of phosphatidylinositol 3-kinase by epidermal growth factor. Int J Radiat Biol 14: 3550-3558, 1994

72. Marone R, Cmiljanovic V, Giese B and Wymann MP: Targeting phosphoinositide 3-kinase - moving towards therapy. Biochim Biophys Acta 1784: 159-185, 2008.

73. Toulany M, Baumann M and Rodemann HP: Stimulated PI3K-AKT signaling mediated through ligand or radiationinduced EGFR depends indirectly, but not directly, on constitutive K-Ras activity. Mol Cancer Res 5: 863-872, 2007.

74. Minjgee M, Toulany M, Kehlbach R, Giehl K and Rodemann HP. K-RAS(V12) induces autocrine production of EGFR ligands and mediates radioresistance through EGFR-dependent Akt signaling and activation of DNA-PKcs. Int J Radiat Oncol Biol Phys 81: 1506-1514, 2011.

75. Toulany M, Lee K-J, Fattah KR, et al: Akt promotes postirradiation survival of human tumor cells through initiation, progression, and termination of DNA-PKcs-dependent DNA double-strand break repair. Mol Cancer Res 10: 945-957, 2012.
76. Sahlberg SH, Gustafsson AS, Pendekanti PN, Glimelius B and Stenerlow B: The influence of AKT isoforms on radiation sensitivity and DNA repair in colon cancer cell lines. Tumour Biol 35 3525-3534, 2014.

77. Shimura T, Kakuda S, Ochiai Y, Kuwahara Y, Takai Y and Fukumoto M: Targeting the AKT/GSK3ß/cyclin D1/Cdk4 survival signaling pathway for eradication of tumor radioresistance acquired by fractionated radiotherapy. Int J Radiat Oncol Biol Phys 80: 540-548, 2011.

78. Kim IA, Bae SS, Fernandes A, et al: Selective inhibition of Ras, phosphoinositide 3 kinase, and Akt isoforms increases the radiosensitivity of human carcinoma cell lines. Cancer Res 65 : 7902-7910, 2005.

79. Contessa JN, Hampton J, Lammering G, et al: Ionizing radiation activates Erb-B receptor dependent Akt and p70 S6 kinase signaling in carcinoma cells. Oncogene 21: 4032-4041, 2002.

80. Kastan MB, Onyekwere O, Sidransky D, Vogelstein B and Craig RW: Participation of p53 protein in the cellular response to DNA damage. Cancer Res 51: 6304-6311, 1991.

81. Shonai T, Adachi M, Sakata K, et al: MEK/ERK pathway protects ionizing radiation-induced loss of mitochondrial membrane potential and cell death in lymphocytic leukemia cells. Cell Death Differ 9: 963-971, 2002.

82.Lee YJ, Soh JW, Jeoung DI, et al: PKC epsilon-mediated ERK $1 / 2$ activation involved in radiation-induced cell death in NIH3T3 cells. Biochim Biophys Acta 1593: 219-229, 2003.

83. Dai X-F, Ding J, Zhang R-G, Ren J-H, Ma C-MC and Wu G: Radiosensitivity enhancement of human hepatocellular carcinoma cell line SMMC-7721 by sorafenib through the MEK/ERK signal pathway. Int J Radiat Biol 89: 1, 2013.

84. Sancar A, Lindsey-Boltz LA, Unsal-Kacmaz K and Linn S Molecular mechanisms of mammalian DNA repair and the DNA damage checkpoints. Annu Rev Biochem 73: 39-85, 2004.

85. Smits VA and Medema RH: Checking out the G(2)/M transition. Biochim Biophys Acta 1519: 1-12, 2001.

86. O'Connell MJ and Cimprich KA: G2 damage checkpoints: what is the turn-on? J Cell Sci 118: 1-6, 2005.

87. Chen T, Stephens PA, Middleton FK and Curtin NJ: Targeting the $\mathrm{S}$ and $\mathrm{G} 2$ checkpoint to treat cancer. Drug Discov Today 17 194-202, 2012

88. Kharbanda S, Saleem A, Datta R, Yuan ZM, Weichselbaum R and Kufe D: Ionizing radiation induces rapid tyrosine phosphorylation of p34cdc2. Cancer Res 54: 1412-1414, 1994.

89. Rhind N, Furnari B and Russell P: Cdc2 tyrosine phosphorylation is required for the DNA damage checkpoint in fission yeast. Genes Dev 11: 504-511, 1997.

90. O'Connell MJ, Raleigh JM, Verkade HM and Nurse P: Chk1 is a weel kinase in the G2 DNA damage checkpoint inhibiting cdc2 by Y15 phosphorylation. EMBO J 16: 545-554, 1997.

91. Lundgren K, Walworth N, Booher R, Dembski M, Kirschner M and Beach D: mik1 and weel cooperate in the inhibitory tyrosine phosphorylation of cdc2. Cell 64: 1111-1122, 1991

92. Parker LL, Atherton-Fessler S and Piwnica-Worms H: p107weel is a dual-specificity kinase that phosphorylates $\mathrm{p} 34 \mathrm{cdc} 2$ on tyrosine 15. Proc Natl Acad Sci USA 89: 2917-2921, 1992.

93. Bulavin DV, Higashimoto Y, Demidenko ZN, et al: Dual phosphorylation controls Cdc25 phosphatases and mitotic entry. Nat Cell Biol 5: 545-551, 2003.

94. Kastan MB and Bartek J: Cell-cycle checkpoints and cancer. Nature 432: 316-323, 2004

95. Nikjoo H, O'Neill P, Wilson WE and Goodhead DT: Computational approach for determining the spectrum of DNA damage induced by ionizing radiation. Radiat Res 156: 577-583, 2001.

96. Yu H: Typical cell signaling response to ionizing radiation DNA damage and extranuclear damage. Chin J Cancer Res 24 83-89, 2012

97. Ward JF: DNA damage as the cause of ionizing radiationinduced gene activation. Radiat Res 138: S85-S88, 1994

98. Haddy N, Tartier L, Koscielny S, et al: Repair of ionizing radiation-induced DNA damage and risk of second cancer in childhood cancer survivors. Carcinogenesis: Apr 19, 2014 (Epub ahead of print).

99. Huhn D, Bolck HA and Sartori AA: Targeting DNA doublestrand break signalling and repair: recent advances in cancer therapy. Swiss Med Wkly 143: w13837, 2013.

100. Hosoya $\mathrm{N}$ and Miyagawa K: Targeting DNA damage response in cancer therapy. Cancer Sci 105: 370-388, 2014

101. Iyama T and Wilson Iii DM: DNA repair mechanisms in dividing and non-dividing cells. DNA Repair 12: 620-636, 2013. 